\title{
New data support the existence of the Hercules-Corona Borealis Great Wall ${ }^{\star}$
}

\author{
István Horváth ${ }^{1}$, Zsolt Bagoly ${ }^{2}$, Jon Hakkila ${ }^{3}$, and L. V. Tóth ${ }^{2}$ \\ 1 National University of Public Service, 1441 Budapest, Hungary \\ e-mail: Horvath. Istvan@uni-nke.hu \\ 2 Eötvös University, 1053 Budapest, Hungary \\ 3 University of Charleston South Carolina at the College of Charleston, Charleston, SC 29424, USA
}

Received 18 August 2014 / Accepted 26 September 2015

\begin{abstract}
Context. Several large structures, including the Sloan Great Wall, the Huge Large Quasar Group, and a large gamma-ray burst cluster referred to as the Hercules-Corona Borealis Great Wall, appear to exceed the maximum structural size predicted by Universal inflationary models. The existence of very large structures such as these might necessitate cosmological model modifications.

Aims. Gamma-ray bursts are the most luminous sources found in nature. They are associated with the stellar endpoints of massive stars and are found in and near distant galaxies. Since they are viable indicators of the dense part of the Universe containing normal matter, the spatial distribution of gamma-ray bursts can serve as tracers of Universal large-scale structure.

Methods. An increased sample size of gamma-ray bursts with known redshift provides us with the opportunity to validate or invalidate the existence of the Hercules-Corona Borealis Great Wall. Nearest-neighbour tests are used to search the larger sample for evidence of clustering and a bootstrap point-radius method is used to estimate the angular cluster size. The potential influence of angular sampling biasing is studied to determine the viability of the results.

Results. The larger gamma-ray burst database further supports the existence of a statistically significant gamma-ray burst cluster at $1.6 \leq z<2.1$ with an estimated angular size of 2000-3000 Mpc.

Conclusions. Although small number statistics limit our angular resolution and do not rule out the existence of adjacent and/or lineof-sight smaller structures, these structures must still clump together in order for us to see the large gamma-ray burst cluster detected here. This cluster provides support for the existence of very large-scale universal heterogeneities.
\end{abstract}

Key words. gamma rays: general - methods: data analysis - methods: statistical - large-scale structure of Universe cosmology: observations - distance scale

\section{Introduction}

The high luminosities of gamma-ray bursts (GRBs) make them ideal candidates for probing large-scale Universal structure. Gamma-ray bursts signify the presence of stellar endpoints and thus trace the location of matter in the universe. This is true whether they are long bursts (presumably originating from hypernovae), short bursts (presumably originating from compact objects), or intermediate bursts (with unknown origins that are still likely related to stellar endpoints). Assuming that the Universe is homogeneous and isotropic on a large scale implies that the large-scale distribution of GRBs should similarly be homogeneous and isotropic. The angular isotropy of GRBs has been well-studied over the past few decades (Briggs et al. 1996; Balázs et al. 1998, 1999; Mészáros et al. 2000; Magliocchetti et al. 2003; Vavrek et al. 2008). For the most part, GRBs are distributed uniformly, although some subsamples (generally believed to be those with lower luminosities and therefore thought to be cosmologically local) appear to deviate from isotropy (Balázs et al. 1998; Cline et al. 1999; Mészáros et al. 2000; Litvin et al. 2001; Magliocchetti et al. 2003; Vavrek et al. 2008). We have recently identified a surprisingly large anisotropy in the overall GRB angular distribution, suggestive of clustering, at redshift two in the constellations of Hercules and Corona

\footnotetext{
$\star$ Appendix $\mathrm{A}$ is available in electronic form at http: //www . aanda.org
}

Borealis. The underlying distribution of matter suggested by this cluster is large enough to question standard assumptions about the largest scale of Universal structures.

We revisit the angular and radial distributions of GRBs with known redshifts in an attempt to reexamine our previous claims suggesting the existence of this structure. As of November 2013, the redshifts of 361 GRBs have been determined $^{1}$; this represents an increase in sample size of $28 \%$ over that used in our previous analysis (283 bursts observed up until July 2012). The number of GRBs in the $z=2$ redshift range, where the cluster resides, has increased from 31 bursts to 44 bursts, a $42 \%$ sample size increase that is large enough to warrant an updated analysis. We apply $k$ th nearest neighbour analysis and the bootstrap point radius method to this database composed largely of bursts detected by NASA's Swift experiment.

\section{Nearest-neighbour statistics: the largest anisotropy is at $z=2$}

The larger GRB database allows us to re-examine the significance of our prior results. We employ the same statistical tests so as to retain consistency in our methodology and not introduce any potential analysis biases.

\footnotetext{
1 http://lyra.berkeley.edu/grbox/grbox.php or http:// www. astro.caltech. edu/grbox/grbox.php
} 
The GRB sample is subdivided by redshift $z$ in a manner similar to our previous work (Horváth et al. 2014) so that we can base our angular studies on well-defined distance groupings. The GRB redshift uncertainties are small (many GRB redshifts are quoted to three or four significant figures), so it is possible in theory to create a large number of radial groups or bins and thus maintain a small $z$-dispersion in the sample. The drawback to this approach is that the 361 burst sample is still small, and angular resolution is limited based on the number of bursts in each radial group. We have subdivided the total sample into eight separate cases, containing the following numbers of radial groups: two, three, four, five, six, seven, eight, and nine. These choices allow us to examine bulk anisotropies in the GRB distribution over various distance ranges. However, binning the data limits the angular resolution we can realistically obtain within each radial bin: we are capable of finding large anisotropies. These cases are not independent of one another; each contains the same GRB sample binned differently. A choice of one radial group corresponds to the bulk angular distribution of GRBs in the plane of the sky; we do not analyse group one here, since it does not make use of the carefully measured redshifts we employed. In contrast, the choice of nine radial groups provides us with radial bins with the smallest number of bursts per bin $(\approx 40)$ for which we can make reasonable, quantifiable estimates on bulk anisotropies. When choosing between 2 and 9 radial divisions, we keep the numbers of bursts in each radial group identical. The result of this approach is that we exclude GRBs with the smallest redshifts in some cases. For example, in the four group case the closest GRB (with the smallest redshift) was excluded, therefore, each of the four groups contains 90 GRBs $(361=4 \times 90+1)$.

We examine the angular burst distributions of each radial group by independently applying the $k$ th nearest-neighbour statistics to the bursts in each group. As an example of our procedure, we consider the four group case again. For each radial group, we calculate the angular separations between all 90 GRBs. All neighbours of each GRB are identified and ranked as the nearest, second nearest, etc. The 90 nearest neighbour separations are collected into a first distribution, the 90 secondnearest neighbour separations are collected into a second distribution, and the process is repeated with each set of neighbours until the orthogonal set is completed with the 89th distribution being composed of the 89th nearest neighbour (farthest) separations. For each group in the four group case, these 89 nearestneighbour distributions can be compared across the groups using a Kolmogorov-Smirnoff test. As this has been accomplished for the four group case, the same approach can be applied to the available nearest-neighbour distributions for all eight radial groupings (the two group case through the nine group case).

Each of the eight radial groupings indicates that significant anisotropies are present in one specific radial region, as defined by redshift. In other words, most of the $k$ th nearest-neighbour distributions are not significantly different, except those that are close to one specific redshift. The bin containing the largest cluster of GRBs always comes from the redshift range $1.6 \leq z<2.1$, as found in our previous work (Horváth et al. 2013, 2014). Figure 1 shows an example of when the sample is divided into four radial groups. In this case, each group contains $90 \mathrm{GRBs}$ in the redshift ranges $2.68 \leq z<9.4$ (group 1), $1.61 \leq z<2.68$ (group 2), $0.85 \leq z<1.61$ (group 3), and $0 \leq z<0.85$ (group 4). For this example, Table 1 shows the probability that the two distributions are different. Boldface type indicates that the significance of the 31st nearest-neighbour distributions of two groups are different by more than $3 \sigma$. There are no
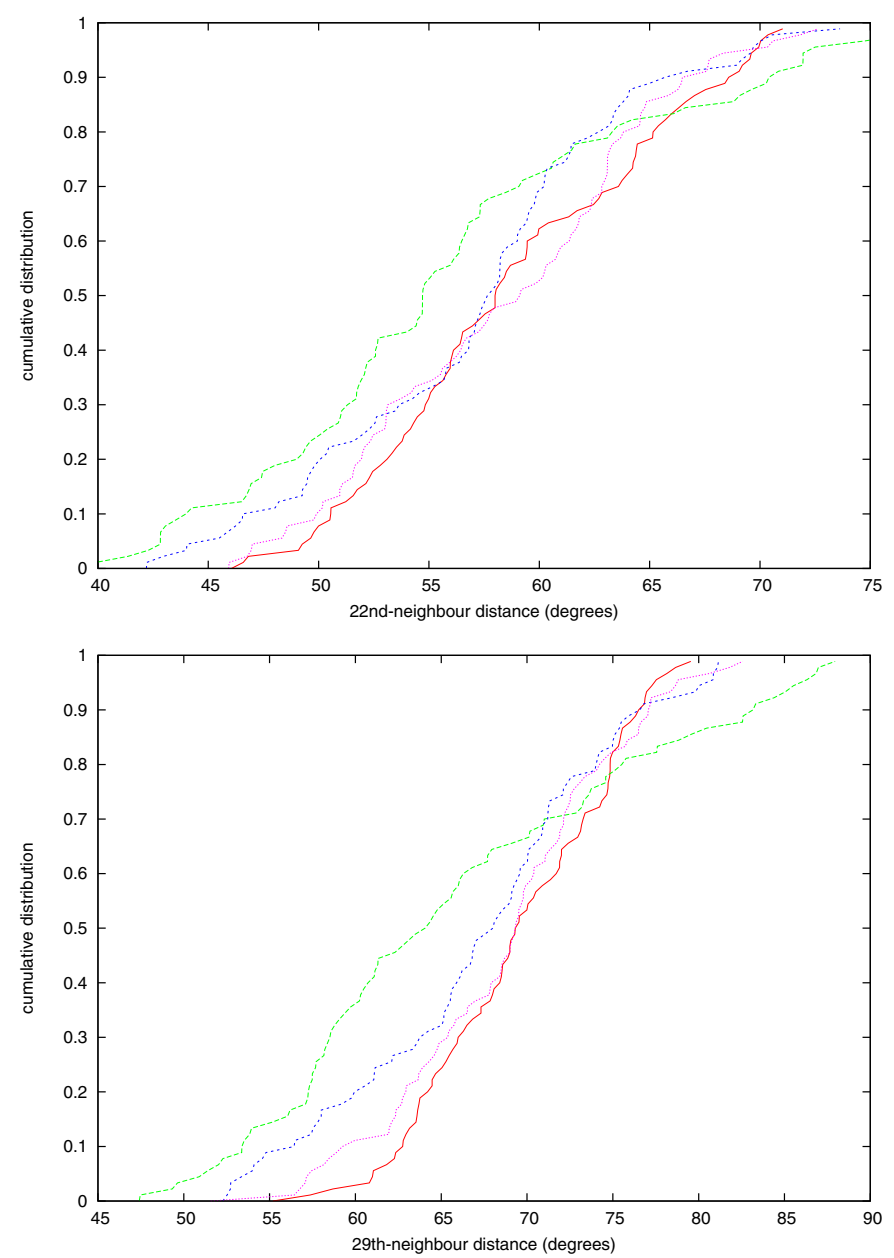

Fig. 1. 22nd (top) and 29th (bottom) neighbour distribution for the four group case (each group contains $90 \mathrm{GRBs}$ ), red, green, blue, and pink identifies radial groups $1-4$.

Table 1. Results of the 31st nearest-neighbour distributions comparing the GRB groups (in the four group case).

\begin{tabular}{ccccc}
\hline \hline & $z_{\min }$ & gr2 & gr3 & gr4 \\
\hline gr1 & 2.68 & $\mathbf{0 . 9 9 9 9 9 9 9}$ & 0.942 & 0.672 \\
\hline gr2 & 1.61 & & $\mathbf{0 . 9 9 9 0 4}$ & $\mathbf{0 . 9 9 9 9 9 8 8}$ \\
\hline gr3 & 0.85 & & & 0.960 \\
\hline
\end{tabular}

Notes. Comparing the distributions of two groups for the 31st nearestneighbour, the numbers in this table are the significance of the null hypothesis that the two distributions are different. Boldface type indicates that there are significant (more than $3 \sigma$ ) differences between the two groups.

significant differences within group 1, group 3, and group 4 distributions, but the 31st nearest-neighbour distribution in group 2 is significantly different than the distributions found in the other groups. The 31st nearest-neighbour distribution is just an example demonstrating a group 2 anisotropy; the same is also true for the 22nd, 23rd, ... 55th nearest-neighbour distributions. The GRBs clustering on small angular scales would show differences when describing close neighbour pairs, while GRBs found on opposite sides of the celestial sphere would exhibit differences when describing distant neighbour pairs. The GRBs in the $1.61 \leq z<2.68$ redshift range have a preference for neighbours with moderately close angular separations, suggesting a large angular cluster. 


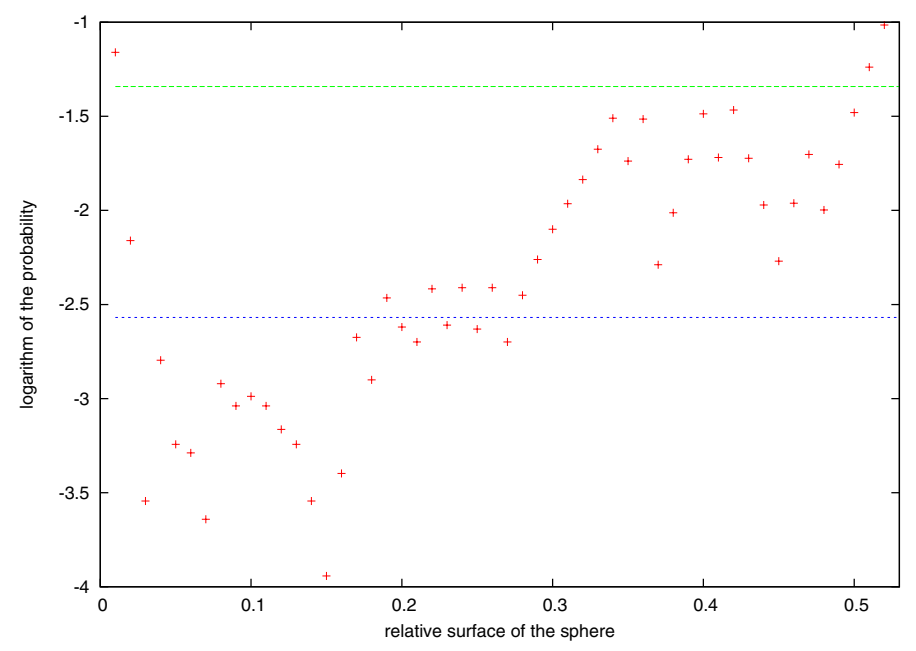

Fig. 2. Results of the Monte-Carlo bootstrap point-radius method. The horizontal coordinate is the area of the circle in the sky relative to the whole sky $(4 \pi)$. The vertical coordinate is the logarithm of the frequency found from the 17500 runs. Green (blue) line shows the $2 \sigma(3 \sigma)$ deviations.

\section{Bootstrap point-radius method: the anisotropy represents a large GRB cluster}

As demonstrated in the previous section, nearest-neighbour tests identify pairing consistent with a large, loose GRB cluster in the redshift range $1.6<z \leq 2.1$. The significance of this cluster can also be measured using other statistical tests designed to identify clustering. Among these is the bootstrap point-radius method described in Sect. 5 of Horváth et al. (2014). The updated data set to which we apply this test contains 44 GRB in the redshift interval $1.6<z \leq 2.1$.

Our use of the bootstrap point-radius method assumes that the sky exposure is independent of $z$. To carry out our analysis, we choose 44 GRBs from the observed data set and compare the sky distribution of this subsample with the sky distribution of 44 GRBs with $1.6<z \leq 2.1$.

To study the selected bursts in two dimensions, we select random locations on the celestial sphere and find how many of the 44 points lie within a circle of predefined angular radius, for example, within $20^{\circ}$. We build statistics for this test by repeating the process a large number of times (i.e., 10000). From the 10000 Monte Carlo runs, we select the largest number of bursts found within the angular circle.

This analysis can be performed with the clustered 44 GRB positions as well as with 44 randomly chosen GRB locations from the observed data. There are some angular radii for which the maximum with the 44 GRBs with $1.6<z \leq 2.1$ is significant. We repeat the process with 44 different randomly chosen burst positions, and we repeated the experiment 17500 times to understand the statistical variations of this subsample. We also perform the same measurement using angular circles of different radii. The frequencies obtained are shown in Fig. 2.

Figure 2 demonstrates that the $9-18 \%$ of the sky identified for $1.6<z \leq 2.1$ contains significantly more GRBs than similar circles at other GRB redshifts. When the area is chosen to be $0.0375 \times 4 \pi$ (corresponding to an angular radius of $\left.\theta_{\max }=22.3^{\circ}\right), 13$ out of the 44 GRBs lie inside the circle. When the area is chosen to be $0.0875 \times 4 \pi\left(\theta_{\max }=34.4^{\circ}\right), 18$ of 44 GRBs lie inside the circle. When the area is chosen to be $0.1875 \times 4 \pi\left(\theta_{\max }=51.3^{\circ}\right), 25 \mathrm{GRBs}$ out of the 44 lie inside the circle. In this last case, only two out of the 17500 bootstrap cases had 25 or more GRBs inside the circle. This result is, therefore, a statistically significant ( $p=0.0001143$ ) deviation, and the binomial probability for this being random is $p_{\mathrm{b}}=2 \times 10^{-8}$.

The $42 \%$ increase in sample size should have led to a noticeable decrease in significance if the sample represented random sampling. However, in the radii between roughly $4^{\circ}$ and $90^{\circ}$, 49 angular circles contain enough GRBs to exceed the $2 \sigma$ level, compared to 28 found in our previous analysis (Horváth et al. 2014). Additionally, there are 16 angular circles containing enough GRBs to exceed the $3 \sigma$ level (compared to only two in our previously published result), therefore, the evidence has strengthened that these bursts are mapping out some large-scale universal structure.

\section{Sky exposure: sampling biases do not appear to be responsible for the anisotropy}

Observing biases can introduce measurable angular anisotropies in a sample. However, prior results suggest that these biases are unlikely to be responsible for the observed cluster at $z \approx 2$. The largest potential causes of angular biasing are:

- Sky exposure. This is a well-known bias describing favoured detection of GRBs in some angular directions over others. Sky exposure is a function of instrumental response rather than a true source distributional preference; some causes of anisotropic sky exposure include spacecraft pointing and a preferred orbital plane, the avoidance of certain pointing directions such as the Sun or occultation by the Earth.

- Anisotropic measurement of GRB redshifts. GRB redshift measurements are made in the visual/infrared by groundbased telescopes, and are thus affected by observatory latitudes, seasonal weather, and Galactic extinction.

Each GRB instrument samples the sky differently, making the summed sky exposure difficult to identify for our heterogeneous GRB sample, which has been observed by many instruments since the late 1990s. However, since more than 3/4 of our sample was detected by Swift, the Swift sky exposure dominates the sampling. Thus, we assume to first order that Swift's sky exposure is a reasonable approximation of the sky exposure of the entire burst sample. Because of its orbital characteristics, Swift (Baumgartner et al. 2013) has sampled ecliptic polar regions at slightly higher rates than ecliptic equatorial regions. Our simple model assumes that ecliptic polar regions $\left(|\beta| \geq 45^{\circ}\right.$, where $\beta$ is the ecliptic latitude) are sampled 1.83 times more frequently than the ecliptic equatorial region.

The location of ground-based optical and infrared telescopes measuring GRB redshifts can also lead to anisotropic observations. However, since a large number of ground-based telescopes at a variety of latitudes and longitudes have been used in GRB follow-up observations, there does not appear to be an Earthbased bias that would favour GRB afterglow measurements in some sky locations over others. Thus, our sampling model does not include a term accounting for telescope location.

Extinction due to dust from the Milky Way disk does not affect the detection of GRBs, but it does affect redshift measurements in an angularly-dependent way. Extinction removes light from extragalactic sources, making it harder to measure spectral characteristics from which redshifts can be obtained. Although the Galactic dust is strongly concentrated towards the Galactic equator, it is also very clumpy. This clumpiness makes the effect of extinction on measuring GRB redshifts very difficult to model; the details of the process depends on many variables, 


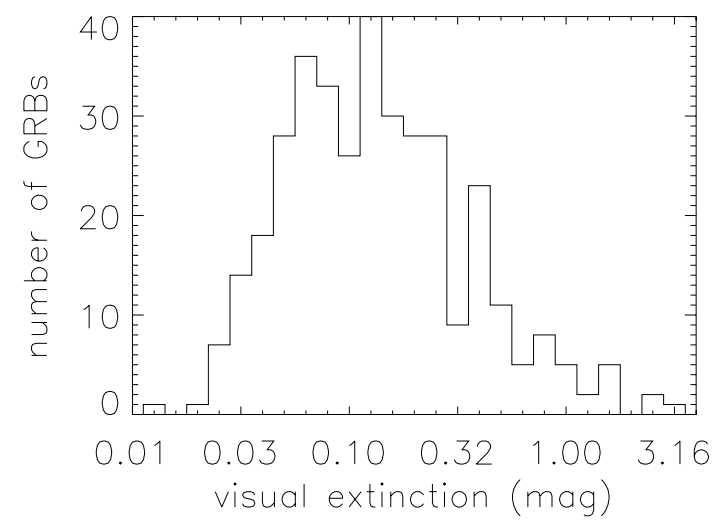

Fig. 3. Galactic visual extinctions in the directions of the $361 \mathrm{GRBs}$ in this sample. The measurement of GRB redshifts strongly favours small amounts of interstellar extinction.

such as the Galactic latitude and longitude of the burst, the intrinsic luminosity and decay rate of the afterglow, the light-gathering ability of the telescope and the instrumental response of the spectrograph used, the redshift of the burst, and the observing conditions at the time of detection.

We check to see whether or not the GRB sample favours low-extinction regions by examining the distribution of visual extinctions in the directions of these 361 bursts. Extinctions are obtained from the high angular resolution DIRBE catalogue of Schlafly \& Finkbeiner $(2011)^{2}$. The results, shown in Fig. 3, are that the sample can be modelled by a lognormal distribution centred at $A_{v}=0.13 \mathrm{mag}$ with standard deviations $\sigma^{+}=0.22$ and $\sigma^{-}=0.08$. Fully $91 \%$ of the bursts in the sample have visual extinctions of $A_{v} \leq 0.5 \mathrm{mag}$, indicating that a characteristic of a GRB with a measured redshift is that it is not obscured by Galactic extinction.

It is not possible to tell if, in trying to measure GRB redshifts from afterglows, observers have avoided pointing their optical telescopes in the direction of GRBs that lie too close to the Galactic equator. This kind of a bias is possible given the potential low return on afterglow detection (especially for small- and medium-sized telescopes), but this bias cannot be modelled with the limited information provided by optical observers. Since the development of a model describing extinction and extinctionrelated biases on redshift measurement is very difficult, to estimate the effects of extinction on the sample we use all GRBs with measured redshifts found within $20^{\circ}$ of the Galactic equator relative to all GRBs with measured redshifts. We find that only $3.1 \%$ of the GRB sample has had redshift measurements made within $20^{\circ}$ of the Galactic equator.

The Galactic equatorial region is the poorest-sampled region. The ecliptic polar regions $\left(b \geq 20^{\circ}\right.$ and $|\beta| \geq 45^{\circ}$ are the bestsampled regions and the ecliptic equatorial regions $\left(b \geq 20^{\circ}\right.$ and $|\beta|<45^{\circ}$ ) are well-sampled regions. Correcting the observations for this biasing, the expected numbers of GRBs in each group are 7.50 (best, north), 7.50 (best, south), 11.2 (good, north), 11.2 (good, south), and 5.54 (poor), whereas the actual counts are 13 (best, north), 2 (best, south), 12 (good, north), 8 (good, south), and 7 (poor). This correction results in a $\chi^{2}$ probability of $p=0.051$ that this is due to chance.

Although this probability is higher than that quoted previously ( $p=0.025)$, the cluster density has increased relative to the rest of the $z \approx 2$ sky since the last analysis. Many

\footnotetext{
2 Found at http://irsa.ipac.caltech.edu/applications/ DUST/
}

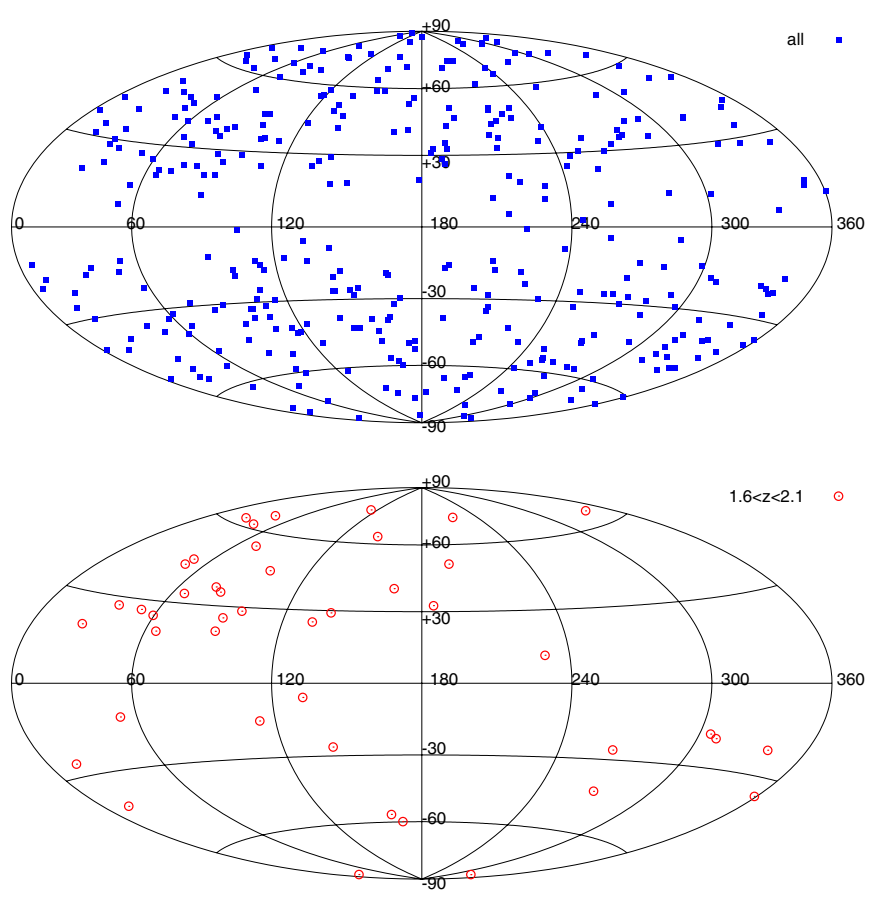

Fig. 4. Top: distribution of GRBs with measured redshift (blue). Although the distribution of all GRBs is fairly isotropic, extinction causes this sample to miss GRBs near the Galactic plane. Bottom: anisotropic distribution of GRBs near redshift $z=2$ (red).

of the new bursts have been detected just outside the edge of the best-sampled region, in the northern well-sampled region. Unfortunately, our low-resolution angular bias correction treats all GRBs in the well-sampled region as if they are not part of the cluster. If we naively assume that the cluster comprises $17 \mathrm{ob}-$ served bursts (13 in $50 \%$ of the northern best-sampled region and 4 in $10 \%$ of the northern well-sampled region) and recalculate the probability that this clustering is random, the probability changes to $p=1.6 \times 10^{-4}$ that exposure is responsible for the clustering. This calculation also suggests that the cluster properties might be affected slightly by exposure: the few bursts seen in regions with less exposure could represent a larger number of undetected bursts. The cluster might be shifted several degrees west of where we have previously identified it.

\section{Summary and conclusion}

The evidence for a possible large-scale Universal structure (Horváth et al. 2014) at a redshift of $z \approx 2$ has strengthened, using a larger database of GRBs with known redshift. The new sample contains $28 \%$ more bursts than the previous sample, and $42 \%$ more bursts in the $1.6 \leq z<2.1$ redshift range. Because the cluster has become more populated relative to the rest of the angular distribution at the same redshift, our angular tests have returned more significant results. Nearestneighbour tests indicate that GRBs in this redshift range favour each others' presence through moderate angular separations. The two-dimensional bootstrap point-radius method reaches the $3 \sigma$ level for a number of different angular radii, indicating a large GRB cluster. Although sampling biases are present and are significant, these coupled with small number statistics, do not seem to be responsible for the observed clustering of GRBs at this redshift. 
GRBs are the most luminous known form of energy release available to normal matter. As such, they are tracers for the presence of normal matter that can be detected at distances where the matter is otherwise too faint to be observed. The GRB cluster at $z \approx 2$ appears to identify the presence of a larger angular structure that covers almost one-eighth of the sky. This encompasses half of the constellations of Bootes, Draco, and Lyra, and all of the constellations of Hercules and Corona Borealis. This structure has been given the popular name of the Hercules-Corona Borealis Great Wall, or Her-CrB GW.

We estimate the size of the Her-CrB GW to be about 2000$3000 \mathrm{Mpc}$ across. Few limits on its radial thickness exist, other than because it appears to be confined to the $1.6 \leq z<2.1$ redshift range. This large size makes the structure inconsistent with current inflationary Universal models because it is larger than the roughly $100 \mathrm{Mpc}$ limit thought to signify the End of Greatness at which large-scale structure ceases.

However, the Her-CrB GW is not the first optical/infrared structure found to exceed the $100 \mathrm{Mpc}$ size limit. Several large filamentary structures have been identified using optical and infrared redshifts of galaxies; these include the $200 \mathrm{Mpc}$-sized CfA2 Great Wall (Geller \& Huchra 1989) and the 400 Mpcsized Sloan Great Wall (Gott et al. 2005). In the ensuing years, other structures have been identified using quasars; the largest of these is the Huge Large Quasar Group (Huge-LQG) Clowes et al. (2013), which has a length of more than $1400 \mathrm{Mpc}$. Most recently, Szapudi et al. (2015) found a 440 Mpc diameter supervoid aligned with a cold spot on the cosmic microwave background.

On the other hand, many results support the cosmological principle. Yahata et al. (2005) reported that the galaxy distribution was homogeneous on scales larger than $60-70 h^{-1} \mathrm{Mpc}$. Bagla et al. (2008) showed that the fractal dimension makes a rapid transition close to 3 at $40-100$ Mpc scales. Sarkar et al. (2009) found the galaxy distribution to be homogeneous at length-scales greater than $70 h^{-1} \mathrm{Mpc}$, and Yadav et al. (2010) estimated the homogeneity upper limit scale was close to $260 h^{-1} \mathrm{Mpc}$.

As large as it appears to be, the Her-CrB GW does not necessarily have to violate the basic assumptions of the cosmological principle (the assumptions of a homogeneous and isotropic Universe). Theoretical large-scale structure models indicate that some structures will exceed the End of Greatness on purely statistical grounds (Nadathur 2013), and this may be one such structure (albeit a very large one). Along these lines, this may not be a single structure, but a clustering of smaller adjacent and/or lineof-sight structures; the small number of bursts currently found in the cluster limits our ability to angularly resolve it. However, this becomes a semantic issue at some point, since a cluster of smaller structures is still a larger structure.

Acknowledgements. This research was supported by OTKA grant NN111016 and by NASA EPSCoR grant NNX13AD28A. We thank the anonymous referee for comments that greatly improved this paper.

\section{References}

Bagla, J. S., Yadav, J., \& Seshadri, T. R. 2008, MNRAS, 390, 829 Balázs, L. G., Mészáros, A., \& Horváth, I. 1998, A\&A, 339, 1 Balázs, L. G., Mészáros, A., Horváth, I., \& Vavrek, R. 1999, A\&AS, 138, 417 Baumgartner, W. H., Tueller, J., Markwardt, C. B., et al. 2013, ApJS, 207, 19 Briggs, M. S., Paciesas, W. S., Pendleton, G. N., et al. 1996, ApJ, 459, 40

Cline, D. B., Matthey, C., \& Otwinowski, S. 1999, ApJ, 527, 827

Clowes, R. G., Harris, K. A., Raghunathan, S., et al. 2013, MNRAS, 429, 2910 Geller, M. J., \& Huchra, J. P. 1989, Science, 246, 897

Gott, III, J. R., Jurić, M., Schlegel, D., et al. 2005, ApJ, 624, 463

Horváth, I., Hakkila, J., \& Bagoly, Z. 2013, 7th Huntsville Gamma-Ray Burst Symposium, GRB 2013: paper 33 in eConf Proc. C1304143 [arXiv: 1311.1104]

Horváth, I., Hakkila, J., \& Bagoly, Z. 2014, A\&A, 561, L12

Litvin, V. F., Matveev, S. A., Mamedov, S. V., \& Orlov, V. V. 2001, Astron. Lett., 27,416

Magliocchetti, M., Ghirlanda, G., \& Celotti, A. 2003, MNRAS, 343, 255

Mészáros, A., Bagoly, Z., Horváth, I., Balázs, L. G., \& Vavrek, R. 2000, ApJ, 539,98

Nadathur, S. 2013, MNRAS, 434, 398

Sarkar, P., Yadav, J., Pandey, B., \& Bharadwaj, S. 2009, MNRAS, 399, L128

Schlafly, E. F., \& Finkbeiner, D. P. 2011, ApJ, 737, 103

Szapudi, I., Kovács, A., Granett, B. R., et al. 2015, MNRAS, 450, 288

Vavrek, R., Balázs, L. G., Mészáros, A., Horváth, I., \& Bagoly, Z. 2008, MNRAS, 391, 1741

Yadav, J. K., Bagla, J. S., \& Khandai, N. 2010, MNRAS, 405, 2009

Yahata, K., Suto, Y., Kayo, I., et al. 2005, PASJ, 57, 529 


\section{Appendix A}

Table A.1. ID, duration, coordinates, and redshift of the 361 GRBs as were published at http://lyra.berkeley.edu/grbox/grbox . php.

\begin{tabular}{|c|c|c|c|c|}
\hline GRB & T90 & RA & Dec & $z$ \\
\hline 090429B & 5.5 & 210.66688 & 32.17064 & 9.4 \\
\hline 090423 & 10.3 & 148.88871 & 18.14939 & 8.2 \\
\hline $120923 \mathrm{~A}$ & 27.2 & 303.79492 & 6.22119 & 8 \\
\hline 080913A & 8 & 65.72775 & -25.1295 & 6.7 \\
\hline 060116 & 35 & 84.69283 & -5.43698 & 6.6 \\
\hline 050904 & 225 & 13.71221 & 14.08661 & 6.295 \\
\hline $120521 \mathrm{C}$ & 26.7 & 214.28668 & 42.14478 & 6 \\
\hline 130606A & 276.6 & 249.39662 & 29.7964 & 5.91 \\
\hline 060927 & 22.6 & 329.55008 & 5.36358 & 5.467 \\
\hline 050814 & 65 & 264.18912 & 46.33933 & 5.3 \\
\hline 071025 & 109 & 355.07116 & 31.77858 & 5.2 \\
\hline 050502B & 7 & 142.54192 & 16.99625 & 5.2 \\
\hline 060522 & 69 & 322.93667 & 2.88621 & 5.11 \\
\hline 111008A & 63.46 & 60.451 & -32.70928 & 4.9898 \\
\hline 060510B & 276 & 239.12167 & 78.57 & 4.9 \\
\hline $100302 \mathrm{~A}$ & 17.9 & 195.51542 & 74.59014 & 4.813 \\
\hline $100513 \mathrm{~A}$ & 84 & 169.61129 & 3.62789 & 4.8 \\
\hline 100219A & 18.8 & 154.20217 & -12.56656 & 4.6667 \\
\hline 090205 & 8.8 & 220.91104 & -27.85297 & 4.6497 \\
\hline $120401 \mathrm{~A}$ & 100 & 58.08258 & -17.63569 & 4.5 \\
\hline 000131 & 50 & 93.37917 & -51.94444 & 4.5 \\
\hline 060223A & 11 & 55.2065 & -17.1301 & 4.41 \\
\hline 080916C & 66 & 119.84717 & -56.63833 & 4.35 \\
\hline 080129 & 48 & 105.28404 & -7.84628 & 4.349 \\
\hline 050505 & 60 & 141.76392 & 30.2735 & 4.27 \\
\hline $120712 \mathrm{~A}$ & 14.7 & 169.58846 & -20.03383 & 4.1745 \\
\hline 090516A & 210 & 138.26092 & -11.85428 & 4.109 \\
\hline 060206 & 11 & 202.93092 & 35.051 & 4.059 \\
\hline $100518 \mathrm{~A}$ & 30 & 304.78917 & -24.55456 & 4 \\
\hline 050730 & 155 & 212.07137 & -3.77158 & 3.9693 \\
\hline $120909 \mathrm{~A}$ & 112 & 275.73633 & -59.44836 & 3.93 \\
\hline 060210 & 255 & 57.73904 & 27.02622 & 3.9122 \\
\hline 090519 & 64 & 142.27917 & 0.18031 & 3.85 \\
\hline 081029 & 270 & 346.77233 & -68.15548 & 3.8479 \\
\hline 081228 & 3 & 39.46225 & 30.85292 & 3.8 \\
\hline $120802 \mathrm{~A}$ & 50 & 44.84313 & 13.76867 & 3.796 \\
\hline 050502A & 20 & 202.44304 & 42.67425 & 3.793 \\
\hline 060605 & 15 & 322.1555 & -6.05869 & 3.773 \\
\hline 130408A & 28 & 134.40542 & -32.36081 & 3.758 \\
\hline 60906 & 43.6 & 40.7535 & 39.36164 & 3.6856 \\
\hline 070721B & 340 & 33.13729 & -2.19461 & 3.6298 \\
\hline 090323 & 150 & 190.70954 & 17.05322 & 3.57 \\
\hline 060115 & 142 & 54.03467 & 17.34531 & 3.5328 \\
\hline 0980329 & 15 & 105.65842 & 38.84556 & 3.5 \\
\hline 051028 & 12 & 27.06254 & 47.75256 & 3.5 \\
\hline 061110B & 128 & 323.91833 & 6.87614 & 3.4344 \\
\hline 0971214 & 50 & 179.10979 & 65.21167 & 3.43 \\
\hline 060707 & 68 & 357.07917 & -17.90472 & 3.424 \\
\hline $121201 \mathrm{~A}$ & 85 & 13.46738 & -42.94289 & 3.385 \\
\hline 090313 & 78 & 198.40088 & 8.09717 & 3.375 \\
\hline 030323 & 25.05 & 166.53917 & -21.77033 & 3.372 \\
\hline 080810 & 106 & 356.79375 & 0.31944 & 3.3604 \\
\hline 110818A & 103 & 317.33769 & -63.98119 & 3.36 \\
\hline 061222B & 40 & 105.3525 & -25.86 & 3.355 \\
\hline 050908 & 20 & 20.46146 & -12.95478 & 3.3467 \\
\hline 050319 & 15 & 154.199 & 43.54858 & 3.2425 \\
\hline 060526 & 13.8 & 232.8265 & 0.2847 & 3.2213 \\
\hline 060926 & 8 & 263.93192 & 13.0385 & 3.2086 \\
\hline 080516 & 5.8 & 120.6415 & -26.15933 & 3.2 \\
\hline 091109A & 48 & 309.25754 & -44.15822 & 3.076 \\
\hline 060607A & 100 & 329.71 & -22.49631 & 3.0749 \\
\hline 081028A & 260 & 121.89471 & 2.30808 & 3.038 \\
\hline
\end{tabular}

Table A.1. continued.

\begin{tabular}{|c|c|c|c|c|}
\hline GRB & T90 & RA & Dec & $z$ \\
\hline 080607 & 79 & 194.94671 & 15.91969 & 3.0368 \\
\hline $121217 \mathrm{~A}$ & 778 & 153.71003 & -62.35098 & 3 \\
\hline 090404 & 84 & 239.23967 & 35.51597 & 3 \\
\hline 090715B & 266 & 251.33967 & 44.83897 & 3 \\
\hline 070411 & 101 & 107.33304 & 1.06461 & 2.954 \\
\hline 120118B & 23.26 & 124.871 & -7.18475 & 2.943 \\
\hline 051008 & 16 & 202.87313 & 42.09814 & 2.9 \\
\hline 060306 & 61 & 41.09546 & -2.14833 & 2.9 \\
\hline 050401 & 38 & 247.87008 & 2.18745 & 2.8983 \\
\hline 111107A & 26.6 & 129.47775 & -66.52008 & 2.893 \\
\hline $120404 \mathrm{~A}$ & 38.7 & 235.0095 & 12.88503 & 2.876 \\
\hline $110731 \mathrm{~A}$ & 38.8 & 280.50412 & -28.53717 & 2.83 \\
\hline 050603 & 6 & 39.98705 & -25.18183 & 2.821 \\
\hline $120327 \mathrm{~A}$ & 62.9 & 246.86442 & -29.415 & 2.813 \\
\hline $130427 \mathrm{~B}$ & 27 & 314.89842 & -22.54636 & 2.78 \\
\hline 081222 & 24 & 22.73996 & -34.09486 & 2.77 \\
\hline 091029 & 39.2 & 60.17742 & -55.95556 & 2.752 \\
\hline 090809 & 5.4 & 328.67996 & -0.08384 & 2.737 \\
\hline 060714 & 115 & 227.86021 & -6.56619 & 2.7108 \\
\hline 090726 & 67 & 248.67935 & 72.88467 & 2.71 \\
\hline $121229 \mathrm{~A}$ & 100 & 190.10121 & -50.5943 & 2.707 \\
\hline 050406 & 3 & 34.46792 & -50.1875 & 2.7 \\
\hline 071031 & 180 & 6.40529 & -58.0595 & 2.6918 \\
\hline 080603B & 60 & 176.53192 & 68.06111 & 2.6892 \\
\hline $120811 \mathrm{C}$ & 26.8 & 199.68254 & 62.30075 & 2.671 \\
\hline 030429 & 9.19 & 183.28125 & -20.91381 & 2.6564 \\
\hline 080210 & 45 & 251.26671 & 13.82669 & 2.6419 \\
\hline 090529 & 100 & 212.469 & 24.45894 & 2.625 \\
\hline 070103 & 19 & 352.5575 & 26.87622 & 2.6208 \\
\hline 050215B & 10 & 174.449 & 40.79581 & 2.62 \\
\hline 050820A & 26 & 337.40879 & 19.56031 & 2.6147 \\
\hline 090426 & 1.2 & 189.07529 & 32.986 & 2.609 \\
\hline $130514 \mathrm{~A}$ & 204 & 296.28292 & -7.97622 & 2.6 \\
\hline 060923A & 51.7 & 254.61733 & 12.36081 & 2.6 \\
\hline 080721 & 16.2 & 224.48273 & -11.72348 & 2.5914 \\
\hline 081118A & 67 & 82.59242 & -43.30147 & 2.58 \\
\hline 050915A & 25 & 81.68668 & -28.01646 & 2.5273 \\
\hline 081121 & 14 & 89.27579 & -60.60286 & 2.512 \\
\hline 050819 & 36 & 358.75675 & 24.86083 & 2.5043 \\
\hline 030115A & 17.94 & 169.63596 & 15.04997 & 2.5 \\
\hline 070529 & 109 & 283.74246 & 20.65944 & 2.4996 \\
\hline $130518 \mathrm{~A}$ & 48 & 355.66781 & 47.46493 & 2.49 \\
\hline $120716 \mathrm{~A}$ & 230 & 313.05042 & 9.59825 & 2.48 \\
\hline 080515 & 21 & 3.1625 & 32.57806 & 2.47 \\
\hline $100424 \mathrm{~A}$ & 104 & 209.44762 & 1.53858 & 2.465 \\
\hline 070802 & 16.4 & 36.89867 & -55.52747 & 2.4541 \\
\hline 090812 & 66.7 & 353.20229 & -10.60472 & 2.452 \\
\hline 071021 & 225 & 340.64296 & 23.71847 & 2.452 \\
\hline 080413A & 46 & 287.299 & -27.67781 & 2.433 \\
\hline 051001 & 190 & 350.95304 & -31.52314 & 2.4296 \\
\hline 080310 & 365 & 220.0575 & -0.17558 & 2.4274 \\
\hline 080905B & 128 & 301.74121 & -62.56306 & 2.3739 \\
\hline $120815 \mathrm{~A}$ & 9.7 & 273.95758 & -52.13114 & 2.358 \\
\hline 070110 & 85 & 0.91363 & -52.97414 & 2.3521 \\
\hline 051109A & 25 & 330.3138 & 40.82314 & 2.346 \\
\hline $110128 \mathrm{~A}$ & 30.7 & 193.89629 & 28.06544 & 2.339 \\
\hline 070129 & 460 & 37.00392 & 11.68444 & 2.3384 \\
\hline 021004 & 52.4 & 6.72783 & 18.92822 & 2.323 \\
\hline 060111A & 13 & 276.205 & 37.60392 & 2.32 \\
\hline 070506 & 4.3 & 347.21829 & 10.72231 & 2.309 \\
\hline $121024 \mathrm{~A}$ & 69 & 70.47208 & -12.29069 & 2.298 \\
\hline $130505 \mathrm{~A}$ & 88 & 137.061 & 17.48478 & 2.27 \\
\hline 081221 & 34 & 15.79258 & -24.54769 & 2.26 \\
\hline 060124 & 710 & 77.10833 & 69.74089 & 2.23 \\
\hline $110205 \mathrm{~A}$ & 257 & 164.62967 & 67.52533 & 2.22 \\
\hline 080804 & 34 & 328.6675 & -53.18461 & 2.2045 \\
\hline
\end{tabular}


Table A.1. continued.

\begin{tabular}{|c|c|c|c|c|}
\hline GRB & T90 & RA & Dec & $z$ \\
\hline $121128 \mathrm{~A}$ & 7.52 & 300.60004 & 54.29978 & 2.2 \\
\hline 050922C & 5 & 317.38785 & -8.75839 & 2.1995 \\
\hline 070810A & 11 & 189.96342 & 10.75119 & 2.17 \\
\hline 071020 & 4.2 & 119.66575 & 32.86111 & 2.1462 \\
\hline 011211 & 270 & 168.82492 & -21.94894 & 2.14 \\
\hline 060604 & 10 & 337.22921 & -10.9155 & 2.1357 \\
\hline 090926A & & 353.40015 & -66.32407 & 2.1062 \\
\hline 100728B & 12.1 & 44.05617 & 0.28106 & 2.106 \\
\hline 060512 & 8.6 & 195.77421 & 41.1909 & 2.1 \\
\hline 081203A & 294 & 233.03158 & 63.52081 & 2.1 \\
\hline $130610 \mathrm{~A}$ & 46.4 & 224.42033 & 28.20711 & 2.092 \\
\hline 061222A & 72 & 358.26425 & 46.53294 & 2.088 \\
\hline 080207 & 340 & 207.51221 & 7.50186 & 2.0858 \\
\hline 000926 & 25 & 256.04046 & 51.78611 & 2.066 \\
\hline 070611 & 12 & 1.99171 & -29.75556 & 2.0394 \\
\hline $000301 \mathrm{C}$ & 10 & 245.0775 & 29.44333 & 2.0335 \\
\hline 060108 & 14.4 & 147.00825 & 31.91906 & 2.03 \\
\hline $130612 \mathrm{~A}$ & 4 & 259.79408 & 16.71997 & 2.006 \\
\hline $121011 \mathrm{~A}$ & 75.6 & 260.21342 & 41.11039 & 2 \\
\hline 080906A & 147 & 228.04438 & -80.51756 & 2 \\
\hline 030226 & 22.09 & 173.27054 & 25.89869 & 1.986 \\
\hline 081008 & 185.5 & 279.95833 & -57.43111 & 1.967 \\
\hline 070419B & 236.5 & 315.70758 & -31.26369 & 1.9588 \\
\hline 050315 & 96 & 306.47542 & -42.60061 & 1.95 \\
\hline 080319C & 34 & 258.98121 & 55.39183 & 1.9492 \\
\hline 060814 & 146 & 221.33871 & 20.58631 & 1.9229 \\
\hline 060708 & 9.8 & 7.80758 & -33.759 & 1.92 \\
\hline 020127 & 7.95 & 123.75592 & 36.77608 & 1.9 \\
\hline 060908 & 19.3 & 31.8265 & 0.342 & 1.8836 \\
\hline 131011A & 77 & 32.52658 & -4.41119 & 1.874 \\
\hline $110801 \mathrm{~A}$ & 385 & 89.43721 & 80.95589 & 1.858 \\
\hline 090902B & 21 & 264.93896 & 27.32419 & 1.822 \\
\hline 090709A & 89 & 289.92767 & 60.72758 & 1.8 \\
\hline $120326 \mathrm{~A}$ & 69.6 & 273.90467 & 69.25986 & 1.798 \\
\hline 080325 & 128.4 & 277.89267 & 36.52342 & 1.78 \\
\hline $121027 \mathrm{~A}$ & 62.6 & 63.59767 & -58.82983 & 1.773 \\
\hline 110422 & 25.9 & 112.04608 & 75.10694 & 1.77 \\
\hline $100425 \mathrm{~A}$ & 37 & 299.1965 & -26.43081 & 1.755 \\
\hline 090113 & 9.1 & 32.0575 & 33.42842 & 1.7493 \\
\hline 120119A & 253.8 & 120.02887 & -9.08158 & 1.728 \\
\hline 100906A & 114.4 & 28.68379 & 55.63044 & 1.727 \\
\hline 050802 & 13 & 219.27371 & 27.78672 & 1.7102 \\
\hline 091020 & 34.6 & 175.72992 & 50.97831 & 1.71 \\
\hline 070521 & 37.9 & 242.66092 & 30.25622 & 1.7 \\
\hline 080928 & 280 & 95.07015 & -55.19971 & 1.6919 \\
\hline 080603A & 180 & 279.40858 & 62.74425 & 1.688 \\
\hline 080605 & 20 & 262.12529 & 4.01556 & 1.6403 \\
\hline 0990510 & 100 & 204.53183 & -80.49689 & 1.619 \\
\hline $110503 \mathrm{~A}$ & 10 & 132.77608 & 52.20753 & 1.613 \\
\hline 0990123 & 63.3 & 231.37642 & 44.76642 & 1.61 \\
\hline 090418A & 56 & 269.31321 & 33.40592 & 1.608 \\
\hline 071003 & 150 & 301.8505 & 10.94772 & 1.6044 \\
\hline 070714A & 2 & 42.93046 & 30.24306 & 1.58 \\
\hline $100728 \mathrm{~A}$ & 198.5 & 88.75838 & -15.25567 & 1.567 \\
\hline 040912 & 150 & 359.179 & -0.92217 & 1.563 \\
\hline 051111 & 47 & 348.13783 & 18.37461 & 1.55 \\
\hline 070125 & 60 & 117.82403 & 31.15114 & 1.5471 \\
\hline 090102 & & 128.24392 & 33.11419 & 1.547 \\
\hline 080520 & 2.8 & 280.19338 & -54.99197 & 1.5457 \\
\hline 060719 & 55 & 18.432 & -48.38092 & 1.532 \\
\hline 030328 & 92.59 & 182.70167 & -9.34758 & 1.522 \\
\hline 080330 & 61 & 169.26873 & 30.6232 & 1.5119 \\
\hline 080805 & 78 & 314.22267 & -62.44439 & 1.5042 \\
\hline 060502A & 33 & 240.927 & 66.60069 & 1.5026 \\
\hline 070306 & 210 & 148.09713 & 10.48202 & 1.49594 \\
\hline 060418 & 52 & 236.4275 & -3.63889 & 1.49 \\
\hline $120724 \mathrm{~A}$ & 72.8 & 245.18062 & 3.50772 & 1.48 \\
\hline
\end{tabular}

Table A.1. continued.

\begin{tabular}{|c|c|c|c|c|}
\hline GRB & T90 & RA & Dec & $z$ \\
\hline 010222 & & 223.05229 & 43.01839 & 1.478 \\
\hline $110213 \mathrm{~A}$ & 48 & 42.96429 & 49.27314 & 1.46 \\
\hline 090407 & 310 & 68.97975 & -12.67922 & 1.4485 \\
\hline 050318 & 32 & 49.71312 & -46.39547 & 1.4436 \\
\hline 100814A & 174.5 & 22.47338 & -17.99544 & 1.44 \\
\hline 050822 & 102 & 51.11342 & -46.03333 & 1.434 \\
\hline 080604 & 82 & 236.96542 & 20.55781 & 1.4171 \\
\hline $100901 \mathrm{~A}$ & 439 & 27.26425 & 22.75856 & 1.408 \\
\hline $120711 \mathrm{~A}$ & 44 & 94.6785 & -70.99911 & 1.405 \\
\hline 080602 & 74 & 19.17571 & -9.23219 & 1.4 \\
\hline $100615 \mathrm{~A}$ & 39 & 177.20542 & -19.48117 & 1.398 \\
\hline 111229A & 25.4 & 76.28692 & -84.71086 & 1.3805 \\
\hline 050801 & 20 & 204.14583 & -21.92806 & 1.38 \\
\hline 090927 & 2.2 & 343.97254 & -70.98036 & 1.37 \\
\hline $100414 \mathrm{~A}$ & 26.4 & 192.11233 & 8.69303 & 1.368 \\
\hline $110808 \mathrm{~A}$ & 48 & 57.26783 & -44.19453 & 1.348 \\
\hline 071117 & 6.6 & 335.04342 & -63.44319 & 1.331 \\
\hline 061121 & 81 & 147.22742 & -13.1952 & 1.3145 \\
\hline 0990506 & 150 & 178.70892 & -26.67644 & 1.307 \\
\hline $130511 \mathrm{~A}$ & 5.43 & 196.64567 & 18.71 & 1.3033 \\
\hline $130420 \mathrm{~A}$ & 123.5 & 196.10654 & 59.42408 & 1.297 \\
\hline 050126 & 26 & 278.11321 & 42.37044 & 1.29 \\
\hline $100724 \mathrm{~A}$ & 1.4 & 194.54333 & -11.1025 & 1.288 \\
\hline 061007 & 75 & 46.33167 & -50.50069 & 1.2622 \\
\hline 020813A & 88.98 & 296.67446 & -19.60134 & 1.2545 \\
\hline 090926B & 81 & 46.30808 & -39.00617 & 1.24 \\
\hline 130907A & 115.1 & 215.892 & 45.60742 & 1.238 \\
\hline 050408 & 34 & 180.57212 & 10.85261 & 1.2356 \\
\hline 080707 & 27.1 & 32.61833 & 33.10953 & 1.2322 \\
\hline $130418 \mathrm{~A}$ & 300 & 149.03717 & 13.66744 & 1.218 \\
\hline 100316B & 3.8 & 163.48812 & -45.47267 & 1.18 \\
\hline 060319 & 12 & 176.38704 & 60.01086 & 1.172 \\
\hline 070208 & 48 & 197.88586 & 61.9651 & 1.165 \\
\hline 070518 & 5.5 & 254.19875 & 55.29508 & 1.16 \\
\hline 061126 & 191 & 86.60198 & 64.21068 & 1.159 \\
\hline 130701A & 4.38 & 357.22954 & 36.10039 & 1.155 \\
\hline 071122 & 68.7 & 276.60525 & 47.07514 & 1.14 \\
\hline 060801 & 0.5 & 213.00554 & 16.98183 & 1.131 \\
\hline 000418 & 30 & 186.33042 & 20.10322 & 1.11854 \\
\hline 0981226 & 260 & 352.40417 & 22.93161 & 1.11 \\
\hline 080413B & 8 & 326.1445 & -19.98111 & 1.1014 \\
\hline 0980613 & 50 & 154.49092 & 71.45708 & 1.0964 \\
\hline 091024 & 1200 & 339.24875 & 56.88983 & 1.092 \\
\hline 110213B & & 41.75588 & 1.14619 & 1.083 \\
\hline 091208B & 14.9 & 29.39204 & 16.88967 & 1.0633 \\
\hline 051006 & 26 & 110.80633 & 9.5068 & 1.059 \\
\hline 000911 & 500 & 34.64317 & 7.74103 & 1.0585 \\
\hline $110726 \mathrm{~A}$ & 5.2 & 286.71692 & 56.07128 & 1.036 \\
\hline 080411 & 56 & 37.97996 & -71.30203 & 1.0301 \\
\hline $121211 \mathrm{~A}$ & 182 & 195.53329 & 30.1485 & 1.023 \\
\hline 0991216 & 50 & 77.38041 & 11.28535 & 1.02 \\
\hline 021211 & 2.8 & 122.24951 & 6.72719 & 1.006 \\
\hline 110918A & 22 & 32.53912 & -27.10544 & 0.982 \\
\hline 071010A & 6 & 288.06093 & -32.40199 & 0.98 \\
\hline 081109 & 190 & 330.7905 & -54.71097 & 0.9787 \\
\hline 091018 & 4.4 & 32.18588 & -57.54828 & 0.971 \\
\hline 120907A & 16.9 & 74.75 & -9.315 & 0.97 \\
\hline 070419A & 116 & 182.74517 & 39.92533 & 0.97 \\
\hline 0980703 & 40 & 359.77779 & 8.5853 & 0.967 \\
\hline $120722 \mathrm{~A}$ & 42.4 & 230.4966 & 13.2513 & 0.9586 \\
\hline 0970828 & 160 & 272.10629 & 59.30236 & 0.958 \\
\hline 071010B & 35.7 & 150.53858 & 45.73064 & 0.947 \\
\hline 071028B & & 354.16167 & -31.62047 & 0.94 \\
\hline 080319B & 50 & 217.92075 & 36.30244 & 0.9382 \\
\hline 060912A & 5 & 5.284 & 20.97161 & 0.937 \\
\hline 051016B & 4 & 132.11583 & 13.65575 & 0.9364 \\
\hline 070714B & 64 & 57.8425 & 28.29761 & 0.923 \\
\hline
\end{tabular}


Table A.1. continued.

\begin{tabular}{|c|c|c|c|c|}
\hline GRB & T90 & RA & Dec & $z$ \\
\hline 090510 & 0.3 & 333.55267 & -26.58411 & 0.903 \\
\hline 070429B & 0.5 & 328.01587 & -38.82833 & 0.9023 \\
\hline 091003 & 21.1 & 251.51953 & 36.62521 & 0.8969 \\
\hline 040924 & 1.2 & 31.594 & 16.11344 & 0.859 \\
\hline $101225 \mathrm{~A}$ & 1088 & 0.19792 & 44.60067 & 0.847 \\
\hline 080710 & 120 & 8.27354 & 19.50147 & 0.8454 \\
\hline 000210 & 20 & 29.81496 & -40.65917 & 0.8452 \\
\hline 0990705 & 45 & 77.47708 & -72.13139 & 0.842 \\
\hline 070318 & 63 & 48.48679 & -42.94619 & 0.84 \\
\hline 0970508 & 35 & 103.45604 & 79.27208 & 0.835 \\
\hline 050824 & 25 & 12.23421 & 22.60922 & 0.8278 \\
\hline 061217 & 0.3 & 160.41383 & -21.12281 & 0.827 \\
\hline $071112 \mathrm{C}$ & 15 & 39.21221 & 28.37131 & 0.8227 \\
\hline $110715 \mathrm{~A}$ & 13 & 237.68371 & -46.23515 & 0.82 \\
\hline 070508 & 21 & 312.80029 & -78.38528 & 0.82 \\
\hline 051022 & 200 & 359.01708 & 19.60669 & 0.809 \\
\hline 100816A & 2.9 & 351.73983 & 26.57858 & 0.804 \\
\hline $120729 \mathrm{~A}$ & 71.5 & 13.07429 & 49.93975 & 0.8 \\
\hline $060602 \mathrm{~A}$ & 60 & 149.56938 & 0.30408 & 0.787 \\
\hline 060202 & 203.7 & 35.84587 & 38.38422 & 0.785 \\
\hline 030528 & 53.85 & 256.00129 & -22.61944 & 0.782 \\
\hline 080430 & 16.2 & 165.31129 & 51.68569 & 0.767 \\
\hline 061110A & 41 & 336.29146 & -2.25886 & 0.7578 \\
\hline 090328 & 80 & 90.66529 & -41.88161 & 0.736 \\
\hline 050813 & 0.6 & 241.98737 & 11.24919 & 0.72 \\
\hline 101219A & 0.6 & 74.58537 & -2.53972 & 0.718 \\
\hline $131004 \mathrm{~A}$ & 1.54 & 296.11283 & -2.95839 & 0.717 \\
\hline 041006 & & 13.70929 & 1.23469 & 0.716 \\
\hline $111228 \mathrm{~A}$ & 101.2 & 150.06671 & 18.29772 & 0.716 \\
\hline 051227 & 8 & 125.24212 & 31.92553 & 0.714 \\
\hline 0991208 & 60 & 248.473 & 46.45583 & 0.7055 \\
\hline 060904B & 192 & 58.2105 & -0.72525 & 0.7029 \\
\hline 090814A & 80 & 239.60979 & 25.63122 & 0.696 \\
\hline 0970228 & 3.6 & 75.44421 & 11.7815 & 0.695 \\
\hline 020405 & 40 & 209.513 & -31.37275 & 0.695 \\
\hline 080916A & 60 & 336.27579 & -57.023 & 0.6887 \\
\hline $111209 \mathrm{~A}$ & 1400 & 14.34492 & -46.80117 & 0.677 \\
\hline 050416A & 2.4 & 188.47747 & 21.0573 & 0.6528 \\
\hline $100418 \mathrm{~A}$ & 7 & 256.36287 & 11.46175 & 0.6235 \\
\hline 110106B & 24.8 & 134.15528 & 47.00291 & 0.618 \\
\hline 070612A & 370 & 121.37337 & 37.27089 & 0.617 \\
\hline 050525A & 10 & 278.13571 & 26.33958 & 0.606 \\
\hline $130215 \mathrm{~A}$ & 65.7 & 43.50292 & 13.39539 & 0.597 \\
\hline 050223 & 23 & 271.38538 & -62.47252 & 0.5915 \\
\hline 060123 & 900 & 179.69933 & 45.51394 & 0.56 \\
\hline 101219B & 34 & 12.23063 & -34.56653 & 0.5519 \\
\hline 051221A & 1.4 & 328.70261 & 16.89088 & 0.5465 \\
\hline 090424 & 52 & 189.52129 & 16.83753 & 0.544 \\
\hline 060729 & 116 & 95.38246 & -62.37022 & 0.5428 \\
\hline $100621 \mathrm{~A}$ & 63.6 & 315.3045 & -51.10625 & 0.542 \\
\hline 090618 & 113.2 & 293.9955 & 78.35686 & 0.54 \\
\hline 081007A & 10 & 339.96 & -40.14689 & 0.5295 \\
\hline 091127 & 7.1 & 36.58288 & -18.95236 & 0.49 \\
\hline 051117B & 8 & 85.18075 & -19.27422 & 0.481 \\
\hline $130831 \mathrm{~A}$ & 32.5 & 358.62458 & 29.42967 & 0.4791 \\
\hline $111211 \mathrm{~A}$ & 15 & 153.09042 & 11.20833 & 0.478 \\
\hline 070724A & 0.4 & 27.80863 & -18.59426 & 0.457 \\
\hline 010921 & 21.77 & 343.99958 & 40.93139 & 0.45 \\
\hline 061006 & 130 & 111.03192 & -79.19864 & 0.4377 \\
\hline 0990712 & 30 & 337.97096 & -73.40786 & 0.43 \\
\hline 020819 & 20 & 351.83112 & 6.26554 & 0.41 \\
\hline 061210 & 85 & 144.52196 & 15.62147 & 0.4095 \\
\hline 120714B & 159 & 355.40875 & -46.18389 & 0.3984 \\
\hline 071227 & 1.8 & 58.13025 & -55.98431 & 0.394 \\
\hline 011121 & & 173.62346 & -76.02819 & 0.362 \\
\hline 130603B & 0.18 & 172.20063 & 17.07167 & 0.356 \\
\hline $110328 \mathrm{~A}$ & & 251.20805 & 57.58325 & 0.354 \\
\hline
\end{tabular}

Table A.1. continued.

\begin{tabular}{lcccc}
\hline \hline GRB & T90 & RA & Dec & $z$ \\
\hline $060428 \mathrm{~B}$ & 58 & 235.35679 & 62.02508 & 0.35 \\
130925A & 0 & 41.179 & -26.1531 & 0.347 \\
061021 & 46 & 145.15058 & -21.95122 & 0.3463 \\
$090417 \mathrm{~B}$ & 260 & 209.69412 & 47.01806 & 0.345 \\
130427A & 162.8 & 173.13683 & 27.69894 & 0.34 \\
050826 & 35 & 87.75658 & -2.64328 & 0.296 \\
$060502 \mathrm{~B}$ & 90 & 278.93971 & 52.63136 & 0.287 \\
$120422 \mathrm{~A}$ & 5.35 & 136.90992 & 14.01875 & 0.283 \\
050724 & 3 & 246.18487 & -27.54097 & 0.258 \\
020903 & 32.15 & 342.17642 & -20.76925 & 0.251 \\
$050509 \mathrm{~B}$ & 0.13 & 189.05858 & 28.98533 & 0.2249 \\
070809 & 1.3 & 203.76896 & -22.14189 & 0.2187 \\
$081211 \mathrm{~B}$ & 102 & 168.26404 & 53.82992 & 0.216 \\
040701 & 60 & 312.06708 & -40.18579 & 0.2146 \\
$030329 \mathrm{~A}$ & 22.76 & 161.20817 & 21.52151 & 0.1687 \\
050709 & 220 & 345.36233 & -38.97764 & 0.16 \\
$130702 \mathrm{~A}$ & 59 & 217.31158 & 15.774 & 0.145 \\
000607 & 0.15 & 38.49475 & 17.14764 & 0.1405 \\
060614 & 102 & 320.88367 & -53.02672 & 0.1257 \\
061201 & 0.8 & 332.13371 & -74.57974 & 0.111 \\
031203 & 30 & 120.6265 & -39.85003 & 0.105 \\
060505 & 4 & 331.76433 & -27.81442 & 0.089 \\
$051109 \mathrm{~B}$ & 15 & 345.45983 & 38.67964 & 0.08 \\
$100316 \mathrm{D}$ & 240 & 107.62642 & -56.25464 & 0.059 \\
060218 & 2100 & 50.41535 & 16.86717 & 0.0331 \\
$111005 \mathrm{~A}$ & 26 & 223.28242 & -19.73672 & 0.01326 \\
0980425 & 30 & 293.76379 & -52.84575 & 0.0085 \\
080109 & 500 & 137.37771 & 33.13897 & 0.006494 \\
\hline
\end{tabular}

